\title{
After the promise: the STD consequences of adolescent virginity pledges
}

\author{
Hannah Brückner, Ph.D. ${ }^{\mathrm{a}}$, and Peter Bearman, Ph.D. ${ }^{\mathrm{b}, *}$ \\ ${ }^{a}$ Department of Sociology, Center for Research on Inequalities and the Life Course, Yale University, New Haven, Connecticut \\ ${ }^{b}$ Department of Sociology, Institute for Social and Economic Research and Policy, Columbia University, New York, New York \\ Manuscript received September 6, 2004; manuscript accepted January 14, 2005
}

\begin{abstract}
Purpose: To examine the effectiveness of virginity pledges in reducing STD infection rates among young adults (ages 18-24).

Methods: Data are drawn from the National Longitudinal Study of Adolescent Health, a nationally representative study of students enrolled in grades 7-12 in 1995. During a follow-up survey in 2001-2002, respondents provided urine samples, which were tested for Human Papilloma Virus, Chlamydia, Gonorrhea, and Trichomoniasis. We report descriptive results for the relationship of pledge status and sexually transmitted disease (STD) rates as well as health behaviors commonly associated with STD infection.

Results: Pledgers are consistently less likely to be exposed to risk factors across a wide range of indicators, but their STD infection rate does not differ from nonpledgers. Possible explanations are that pledgers are less likely than others to use condoms at sexual debut and to be tested and diagnosed with STDs.

Conclusions: Adopting virginity pledges as intervention may not be the optimal approach to preventing STD acquisition among young adults. (C) 2005 Society for Adolescent Medicine. All rights reserved.
\end{abstract}

Keywords: $\quad$ Virginity pledge; Adolescent sexual behavior; STD acquisition; STD testing; STD risk factors

Understanding the determinants of sexually transmitted disease (STD) acquisition among adolescents and young adults is critical to assess interventions designed to limit the spread of STDs [1-3]. One set of interventions-adopted by numerous organizations and directly supported by federal policy-are programs that encourage abstinence by encouraging adolescents to make pledges to remain virgins until marriage. This article considers the relationship between adolescent virginity pledges and the sexual behavior of young adults, focusing on STD acquisition.

In 1993, "True Love Waits" initiated a movement to encourage adolescents to pledge to abstain from sex until marriage. By 1995, an estimated 2.2 million adolescents

\footnotetext{
*Address correspondence to: Dr. Hannah Brückner, Department of Sociology, Yale University, P.O. Box 208265, New Haven, CT 065208265.

E-mail address: hannah.brueckner@yale.edu
}

(12\% of all adolescents) in the United States had taken such pledges. Earlier research demonstrated that adolescent virginity pledges were associated with a significant delay on the baseline rate for the transition to first sex [4]. Although pledging was associated with delayed sexual initiation, the pledge effect was deeply shaped by social context, most importantly, the number of other students in the community that pledged and the social structure of the community with respect to the patterning of friendships. The pledge appears to work where public commitment to abstain from sex is encoded into shared group activities, thus enhancing identification with the movement and encoding the promise to remain a virgin into the larger social fabric.

If taking a pledge indeed reduces premarital sexual activity, one might expect that pledgers are less likely to contract STDs than others because they initiate sexual activity later, have fewer sexual partners, and are more likely to have sex in the context of a marital relationship than 
others, all protective factors. On the other hand, pledgers may be more exposed to infection than others because they may be less likely to use condoms [4]. We explore the sexual and health behaviors that may mediate the relationship between pledging history and later STD status. If young people take a public virginity pledge to remain virgins until marriage, having sex before marriage means that they break their pledge. Thus, sexually active pledgers have a greater incentive than nonpledgers to hide that they are having sex. Especially critical are interactions with health professionals able to provide services to those who suspect they may have an STD, pharmacists and others who provide access to condoms, contraceptive information, and STD counseling, and friends and family members who could provide relevant information about STDs, but may consider such information unnecessary. Against this background, we consider the relationship between pledging and self-reported STD-related health care utilization.

\section{Data and methods}

The initial results on the impact of virginity pledges on the transition to first sex arose from analyses of the first two waves of data from National Longitudinal Study of Adolescent Health (hereafter, Add Health). In this article, we consider data from the 3rd in-home wave of Add Health interviews, when respondents were 18-24 years old. This enables us to consider the long-term consequences of adolescent pledging on the sexual behavior and STD acquisition dynamics of young adults.

Of the original Add Health wave 1 respondents ( $\mathrm{n}=$ 20,745), 15,170 individuals, or 73\%, participated in wave 3 . Data were collected between August 2001 and April 2002. Biomarker data (urine samples) on STD status were collected from $92 \%$ of wave 3 respondents. A total of 1183 individuals $(8 \%)$ refused participation in the biospecimen collection. Urine samples were collected in the field and analyzed for the presence of three sexually transmitted diseases, Chlamydia (CH), Gonorrhea (GC), and Trichomoniasis (TR). In addition, 7000 female respondents who reported ever having had vaginal sex in wave 3 were randomly selected for Human Papilloma Virus (HPV) assays. The specific procedure and tests used are described in detail in [5]. Both males and females were tested for the three bacterial STDs regardless of their sexual activity status.

Collection procedures followed a strict protocol; samples that arrived in a condition not suitable for testing were discarded. Between $4 \%(\mathrm{CH}), 5 \%(\mathrm{TR})$, and $10 \%$ (GC) of the samples were not tested for these reasons $[5,6]$. Pledgers do not differ from others with respect to whether their samples yielded results in the testing ( $p=0.32$ for $\mathrm{CH}, p=$ 0.17 for TR, $p=0.20$ for CG). Pledgers did not differ from others in the extent to which they refused to provide urine samples $(p=0.28)$. Data are weighted to adjust for over- sampling of various groups and wave 3 nonresponse. Analyses of panel attrition for wave 3 show that generally, nonresponse bias has little impact on estimates [7]. If pledgers were significantly more or less likely than others to participate in wave 3 , however, weighting may not correct the resulting bias. Among females, respondents who reported pledging in wave 1 or 2 were just as likely to participate in wave 3 as nonpledgers. Among males, those who pledged in wave 1 or wave 2 were more likely to participate in wave 3 than others (23\% nonresponse compared with 29\%). However, both pledging and participation in wave 3 is negatively associated with age. The difference between pledgers and others is significant only for males who were 17 years and older at the time of wave $1(31 \%$ vs. $22 \%$ nonresponse); among males under age 15 and between 15 and 16 , nonresponse differs by $3 \%$ and $6 \%$, respectively, but not statistically significantly.

Unless otherwise noted, the analyses reported below are based on 11,471 respondents with valid data on STD status and grand sample weights. The majority of the results are derived from cross-tabulating pledge status with various outcome and behavioral measures. To adjust standard errors for the clustered sample design, we used the survey procedures provided in STATA [8,9]. For two measures (timing of first sex and first marriage) we used Kaplan-Meier estimates of the survivor function; these analyses use weights, but do not adjust for clustering. A Wald test based on a robust variance estimator appropriate for weighted data was used to test for the difference between observed and expected number of failures within each group.

\section{Measurement}

Pledge status was collected across all three waves. In wave 1 and 2, respondents were asked "Have you taken a public or written pledge to remain a virgin until marriage?" In wave 3, respondents were asked "Have you ever signed a pledge to abstain from sex until marriage?" We distinguish between individuals who reported that they took a pledge in one of the three waves and later said they did not take a pledge (inconsistent pledgers) and those whose pledge reports are consistent across waves. Respondents who reported pledging for the first time in the wave 3 were classified as consistent pledgers. The third group (nonpledgers) comprises respondents who never reported a pledge $(80 \%, \mathrm{n}=9072)$. Thus, one in five respondents reported pledging in at least one wave, but only $7 \%(\mathrm{n}=777)$ gave consistent answers. The remainder $(13 \%, \mathrm{n}=1622)$ reported a pledge in one wave but said they had not taken a pledge in a later wave. It is unfortunate that the wording of the item was changed in wave 3 ; however, inconsistencies are frequent even when comparing only wave 1 and 2 . About half of those who reported a pledge in wave 1 and participated in wave 2 said they had not taken a pledge in wave 2. Response inconsistency in longitudinal studies is 
relatively common. In general, consistency is greatest when the behavior under investigation is more salient. Considering the complete set of "ever" questions for which Add Health collected data, consistency of pledging appears to be in the middle of the salience distribution, above smoking, but below sexual orientation or school expulsion. Here, we consider all three groups-consistent pledgers, inconsistent pledgers, and nonpledgers.

We examine STD status of the three groups using data from analyses of urine samples for Human Papillomavirus (HPV), Chlamydia (CH), Gonorrhea (GC), and Trichomoniasis (TR) at the time of the wave 3. We hypothesize that inconsistent pledgers will be less exposed to infection risk than nonpledgers but more than consistent pledgers, and therefore have intermediate STD rates. For the less prevalent STDs (GC, $\mathrm{CH}, \mathrm{TR}$ ) we aggregate test results into a single dichotomous indicator to increase sample size. This is justifiable because all three are transmitted through sexual intercourse. HPV is analyzed separately because it is much more prevalent, may be transmitted by noncoital sexual behavior, and the testing was based on a different sample $(n=3317$; sexually active females only).

Sexual and health behavior. The timing of the transition to first sex was measured using self-reported age at first vaginal intercourse. The month and year of this event was taken from wave 1 for those who reported being sexually experienced at that time; from wave 2 for those who transitioned between waves 1 and 2 . For those who transitioned thereafter, we used self-reported age at first vaginal intercourse from wave 3. In wave 3 , respondents were asked for age at first intercourse rather than year and month of the event. In contrast to earlier research, we therefore measure the differences between pledgers and others only in years, rather than months. Marriage age was calculated from the month and year of the first marriage reported in wave 3 .

It was more difficult to determine the timing of pledging because neither wave 1 nor wave 2 included a question about the timing. Although wave 3 contained a retrospective question about the timing of pledging, this question was asked only of respondents who reported having ever taken a pledge in wave 3 . Thus, those who reported pledging in wave 1 or 2 but not in wave 3 were not asked when they had taken a pledge. However, in many cases a time-order between pledging and sexual debut could be determined by comparing reported pledge and virginity status across waves. We were thus able to determine the time-ordering between first sex and pledge for $93 \%$ of pledgers. The pledge movement allows nonvirgins to participate in pledging. We refer to adolescents who pledged after having had intercourse as "secondary virgins." Of those who reported pledging in any wave, $11 \%$ were secondary virgins, $21 \%$ reported no vaginal sex in any wave, and $61 \%$ had sex after pledging; $6 \%$ had missing data or a tie.

Number of sexual partners was measured as the number of partners reported in wave 3 with whom the respondents had vaginal intercourse. We also calculated the number of years a respondent was exposed as difference between age at first vaginal sex and age at interview. To measure STD risk associated with partners, we used a question that asked respondents for each partner "As far as you know, during the time you and $<$ PARTNER $>$ have had a sexual relationship, has < PARTNER $>$ had any other sexual partners?" Similar to our measure of transition to first sex, condom use at first vaginal sex was measured from self-reported data across all 3 waves of data collection. In wave 1 and 2, condom use at first vaginal intercourse was asked directly. For those who reported vaginal sex for the first time in wave 3 , we extracted this information from the relationship information, because condom use was reported for the first vaginal intercourse for each sexual relationship reported by the respondent. Where available, we used the start and ending dates of the sexual relationship to identify the first one. Otherwise, we used a question that asked the respondent to chronologically order all relationships to determine which was the first.

Add Health asked respondents also about their experiences with STDs. Specifically, all respondents were asked whether they had been diagnosed with various STDs in the past year; whether they had ever seen a doctor because they were worried about having a STD; and whether they had been tested for various STDs in the past year.

\section{Results}

\section{STD acquisition}

Bio-marker rates were as follows: TR $(2.3 \%, 95 \% \mathrm{CI}$ $1.8-2.8 \%), \mathrm{CH}(4.2 \%, 95 \%$ CI $3.6-4.9 \%), \mathrm{GC}(0.4 \%, 95 \%$ CI $0.3-0.6 \%)$, HPV $(28.8 \%, 95 \%$ CI $26.3-31.4 \%)$. Although these rates may be somewhat lower than those found in the literature, it bears noting that they are derived from a representative random sample of the population aged 18-24 years. Other studies use clinical samples or special populations and may therefore overestimate STD prevalence [10]. STD acquisition varies significantly by race and ethnicity. Table 1 reports current infection with TR, GC, and/or $\mathrm{CH}$ by race, ethnicity and gender (Panel A). Results for HPV are reported separately (Panel B).

Black males and black females have rates roughly 8 times that for white males and females, respectively. Asian, Hispanic, and others have rates 2 to 5 times that of Whites for TR, GC, and/or $\mathrm{CH}$. The prevalence for HPV varies between $16 \%$ for Asian females, $25 \%$ for white females, $30 \%$ for Hispanic females, and $34 \%$ for black females. Because both race/ethnicity and pledging are associated with STD infection, we consider the effect of the pledge on the likelihood of having an infection within race/ethnic groups. Owing to small sample sizes, we combine the data for Hispanics, Asians, and respondents of other race/ethnic- 
Table 1

STD prevalence rates by race/ethnicity and gender $^{\mathrm{a}}$

\begin{tabular}{|c|c|c|c|c|c|c|c|c|}
\hline & \multicolumn{4}{|l|}{ Female } & \multicolumn{4}{|l|}{ Male } \\
\hline & Percent & $95 \%$ & $\mathrm{CI}$ & $\mathrm{n}$ & Percent & $95 \%$ & $\mathrm{CI}$ & $\mathrm{n}$ \\
\hline \multicolumn{9}{|c|}{ Panel A: GC, TR, and/or $\mathrm{CH}(\%)^{\mathrm{b}}$} \\
\hline White & 3.8 & 3.1 & 4.6 & 3316 & 2.9 & 2.2 & 3.7 & 2926 \\
\hline Black & 24.0 & 20.4 & 28.1 & 1340 & 16.0 & 12.9 & 19.9 & 1041 \\
\hline Hispanic & 6.4 & 4.8 & 8.5 & 923 & 9.7 & 7.1 & 13.2 & 901 \\
\hline Asian & 5.9 & 3.6 & 9.5 & 381 & 5.4 & 2.6 & 10.7 & 427 \\
\hline Other & 15.8 & 7.8 & 29.5 & 105 & 7.0 & 2.7 & 16.6 & 105 \\
\hline Total $^{\mathrm{c}}$ & 7.5 & 6.5 & 8.7 & 6069 & 5.8 & 4.9 & 6.9 & 5402 \\
\hline \multicolumn{9}{|c|}{ Panel B: HPV (Female only, \%) ${ }^{\mathrm{d}}$} \\
\hline White & 25.5 & 23.0 & 28.2 & 1806 & & & & \\
\hline Black & 34.1 & 29.2 & 39.2 & 809 & & & & \\
\hline Hispanic & 29.5 & 23.1 & 36.8 & 519 & & & & \\
\hline Asian & 16.3 & 9.4 & 23.7 & 180 & & & & \\
\hline Other & 23.7 & 12.1 & 41.3 & 53 & & & & \\
\hline Total & 27.2 & 26.3 & 31.4 & 3367 & & & & \\
\hline
\end{tabular}

Standard errors adjusted for clustering.

${ }^{a}$ STD status measured at wave 3; percentages weighted using the longitudinal weight for wave 3 .

${ }^{\mathrm{b}}$ Includes respondents who never reported vaginal sex.

${ }^{\mathrm{c}}$ Includes 6 respondents with missing race/ethnicity.

${ }^{\mathrm{d}}$ Includes only females who reported vaginal sex in wave 3 .

ity, and report HPV separately only for Whites. Table 2 shows that there are no significant differences in STD rates across any of the pledge groups compared with nonpledgers. For most groups, the point estimates are fairly similar as well. With respect to the summary indicator for TR, GC, and $\mathrm{CH}$, the relatively largest differences are found for white respondents, with point estimates that are $31 \%$ lower for inconsistent pledgers and $6 \%$ for consistent pledgers (Panel A). The rates are so low, however, that we cannot reject the null hypothesis of zero difference in the rates. Although the rates are higher for the other groups, the differences are too small to be significant, statistically or substantively. Point estimates for HPV are slightly higher for pledgers (Panel B), albeit not significantly so.
If nonresponse were significantly different for the pledge groups, these findings might be owing to selectivity. For example, nonpledgers at risk for STD might be less likely to participate in wave 3 , which would lead to a downwardly biased estimate for the group. As explained above, no response bias associated with pledge status was found for females. There was differential response for males, specifically older males, however. By comparing STD rates within age groups by pledge status in waves 1 and 2, we can make an informed guess about the significance of response bias. Because of the small cell sizes, here we combine consistent and inconsistent pledgers. If differential response was solely responsible for the findings, we should see lower rates for pledgers among those who were under age 15 at the

Table 2

STD status by pledge status and race/ethnicity ${ }^{\mathrm{a}}$

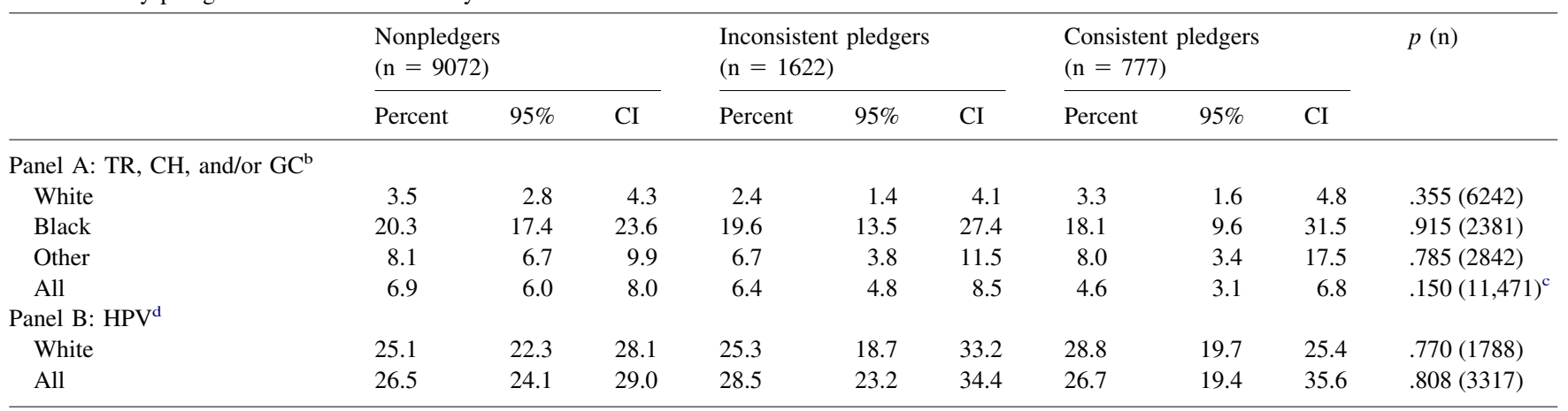

${ }^{\text {a }}$ Pledge status measured across waves 1-3; STD status measured at wave 3; percentages weighted using the longitudinal weight for wave 3. Standard errors adjusted for clustering.

${ }^{\mathrm{b}}$ Includes respondents who never reported vaginal sex.

${ }^{\mathrm{c}}$ Includes 6 respondents with missing race/ethnicity.

${ }^{\mathrm{d}}$ Includes only females who reported vaginal sex in wave 3 . 
time of wave 1 because in this group, both pledgers and others were equally likely to participate in wave 3 . In contrast, among older males, we should see smaller or no differences, because the hypothesis is that in these groups, nonpledgers who are at risk for STD are less likely to participate. Differences are small and not significant in all three age groups, but they are larger among older adolescents $(3.4 \%$ infected pledgers for the TR/CH/GC indicator, compared with $5.9 \%$ for nonpledgers). The differences are smaller in the youngest group $(4.3 \%$ vs. $5.1 \%)$ and the next-youngest group (5.6\% vs. $7.0 \%)$. This pattern is not what we would expect if the lack of a difference between pledgers and others was solely owing to nonresponse associated with pledge status. There are several possible explanations for this finding. Differences between pledgers and others in sexual risk-taking might be smaller than expected, or other risk factors, such as differences in condom use, might be more important. We explore these possibilities in the following.

\section{Sexual debut and marriage}

Table 3 summarizes Kaplan-Meier estimates for the transition to first vaginal sex and first marriage. Cell entries in Table 3 denote the age at which the percentile indicated in each row experienced the event in question. Thus, $25 \%$ of nonpledgers had experienced sexual debut by the time they turned $15,50 \%$ when they turned 17 , and $75 \%$ when they turned 18 (Panel A). Consistent pledgers, in contrast, reach the 25 th percentile by age 17 , the median by age 19 , and the 75 th percentile at age 24 . The inter-quartile difference is 3 years for nonpledgers, 4 years for inconsistent pledgers, and 7 years for consistent pledgers. The Wald test for equality of the survivor functions for the three groups yields a $\chi^{2}$ of 189 for females and 140 for males ( $p \leq .000$ for both tests). Thus, pledgers experience first sex later than others across adolescence, and a significant minority holds out far into young adulthood. By age 25, we estimate that $25 \%$ of consistent male pledgers are still virgins, compared with $7 \%$ of nonpledgers and $15 \%$ of inconsistent pledgers. For females, the corresponding numbers are $21 \%$ for consistent pledgers, $6 \%$ for nonpledgers, and $10 \%$ for inconsistent pledgers. Note that these results are for vaginal intercourse only.

Table 3, panel B shows that female pledgers marry earlier than nonpledgers. The summary measures in Table 3 conceal, however, substantial differences in the timing of marriage. We estimate that by age 25 more than half (52\%) of female consistent pledgers are married, compared with $40 \%$ for inconsistent pledgers and 34\% for nonpledgers. The differences in the survivor functions for females are significant with a $\chi^{2}$ of $14.6(p=.001)$. For males, the corresponding numbers are $45 \%$ for consistent pledgers, compared with $26 \%$ for inconsistent pledgers and $25 \%$ for nonpledgers. The differences in the survivor functions are
Table 3

Transition to first sex and marriage, by pledge status ${ }^{a}$

\begin{tabular}{|c|c|c|c|}
\hline & Nonpledgers & $\begin{array}{l}\text { Inconsistent } \\
\text { pledgers }\end{array}$ & $\begin{array}{l}\text { Consistent } \\
\text { pledgers }\end{array}$ \\
\hline \multicolumn{4}{|c|}{$\begin{array}{l}\text { Panel A: Transition to First } \\
\text { Sex }\end{array}$} \\
\hline \multicolumn{4}{|l|}{ All } \\
\hline $25 \%$ tile & 15 & 16 & 17 \\
\hline Median & 17 & 18 & 19 \\
\hline $75 \%$ tile & 18 & 20 & 24 \\
\hline \multicolumn{4}{|l|}{ Female } \\
\hline $25 \%$ tile & 15 & 16 & 16 \\
\hline Median & 17 & 18 & 18 \\
\hline $75 \%$ tile & 18 & 19 & 23 \\
\hline \multicolumn{4}{|l|}{ Male } \\
\hline $25 \%$ tile & 15 & 16 & 17 \\
\hline Median & 17 & 18 & 20 \\
\hline $75 \%$ tile & 18 & 21 & 25 \\
\hline \multicolumn{4}{|c|}{$\begin{array}{c}\text { Panel B: Age at First } \\
\text { Marriage }\end{array}$} \\
\hline \multicolumn{4}{|l|}{ All } \\
\hline $25 \%$ tile & 24 & 23 & 23 \\
\hline Median & - & - & - \\
\hline \multicolumn{4}{|l|}{ Female } \\
\hline $25 \%$ tile & 23 & 22 & 22 \\
\hline Median & - & - & 24 \\
\hline \multicolumn{4}{|l|}{ Male } \\
\hline $25 \%$ tile & 24 & 24 & 24 \\
\hline Median & - & - & - \\
\hline
\end{tabular}

${ }^{\text {a }}$ Kaplan-Meier estimates for survivor function; cells entries denote age at which the percentile indicated in each row experienced first vaginal sex/first marriage. Data are weighted with longitudinal weight for wave 3 .

not statistically significant for males. Respondents who were married at the time of wave 3 were significantly less likely to test positive for TR/CH/GC (4.1\% vs. $7.2 \%, \mathrm{n}=$ $11,741, p=.0003)$ and HPV (16.3\% vs. $29.6 \%, \mathrm{n}=3317$, $p=.0000)$. Among married respondents, STD rates did not differ by pledge status (data available on request).

Although pledgers tend to have sex later than nonpledgers and tend to get married earlier than nonpledgers, most pledgers do not wait to get married before having first sex. If we consider just those respondents who have had sex, $88 \%$ of the pledgers have sex (here again, vaginal intercourse) before they get married, whereas $99 \%$ of the nonpledgers have sex before marriage. Inconsistent pledgers (94\%) are in between, as expected. Of all respondents, not conditioning on having had sex, the pattern is roughly similar. Specifically, $61 \%$ of all pledgers, $90 \%$ of all nonpledgers, and $79 \%$ of all inconsistent pledgers have sex before marriage or interview date.

\section{Time-order between sexual debut and pledging}

One potentially confounding issue with respect to the interpretation of the pledge effect is the problem of "secondary virgins." The pledge movement makes room for people who have had sex to subsequently take a virginity pledge. Secondary virgins may shape the differences in 
Table 4

Health behavior and virginity pledge $\mathrm{e}^{\mathrm{a}}$

\begin{tabular}{|c|c|c|c|c|c|c|c|c|c|c|}
\hline & \multicolumn{3}{|c|}{ Nonpledgers } & \multicolumn{3}{|c|}{ Inconsistent pledgers } & \multicolumn{3}{|c|}{ Consistent pledgers } & $p \leq$ \\
\hline Self-reported TR/CH/GC & 3.6 & 3.0 & 4.2 & 3.1 & 2.2 & 4.3 & 2.8 & 1.7 & 4.5 & .475 \\
\hline Tested $(\mathrm{TR}, \mathrm{CH}, \mathrm{GC})^{\mathrm{c}}$ & 30.3 & 28.2 & 32.5 & 23.8 & 19.9 & 28.2 & 18.5 & 13.9 & 24.1 & .000 \\
\hline Tested $(\mathrm{TR}, \mathrm{CH}, \mathrm{GC})^{\mathrm{d}}$ & 10.0 & 8.8 & 11.3 & 7.5 & 4.7 & 11.6 & 5.6 & 2.6 & 11.7 & .145 \\
\hline Tested (HPV) ${ }^{\mathrm{b}}$ & 14.9 & 13.6 & 16.3 & 10.3 & 7.9 & 13.2 & 7.8 & 5.8 & 10.3 & .000 \\
\hline Ever seen doctor for $\mathrm{STD}^{\mathrm{e}}$ & 20.8 & 19.3 & 22.3 & 15.1 & 12.9 & 17.6 & 15.9 & 12.4 & 20.2 & .000 \\
\hline Used condom at first sex & 59.7 & 58.0 & 61.4 & 54.9 & 51.2 & 58.2 & 54.6 & 48.5 & 60.0 & .017 \\
\hline
\end{tabular}

Standard errors adjusted for clustering.

${ }^{\text {a }}$ STD status measured at wave 3; Percentages weighted using the longitudinal weight for wave 3.

${ }^{\mathrm{b}}$ Females.

${ }^{\mathrm{c}}$ Females who report any sexual activity in wave 3 .

${ }^{\mathrm{d}}$ Males who report any sexual activity in wave 3 .

${ }^{\mathrm{e}}$ Respondents who report any sexual activity in wave 3 .

transition dynamics for those who are consistent versus inconsistent pledgers. This is not the case. Although slightly more consistent pledgers (14\% rather than $10 \%$ ) are "secondary virgins," this difference is not substantial enough to affect the results reported above.

It is also possible that "secondary virgins" are confounding the substantive interpretation of the results reported in Table 2. For example, some respondents could have had sex, thought they might have contracted an STD, felt bad about that-or felt bad about having sex in general-and subsequently pledged to remain a virgin until marriage. If this were the case, the fact that pledgers are as likely to have an STD as nonpledgers could be an artifact of the timing of pledging and sex. This does not seem to be the case- of those who had sex before they pledged (the secondary virgins) the prevalence rate for TR, GC, and/or $\mathrm{CH}$ is $5.5 \%$. In contrast, $7.3 \%$ of those who pledged before sex have a current STD infection, in contrast to $6.9 \%$ of those who never pledged. If the secondary virgins are playing a role in pledger STD infection rates, it is likely in the opposite direction. Those who have sex and then pledge are less likely to have an STD than those who pledge and then have sex.

\section{Condom use, oral and anal sex}

Condom use at first intercourse is a powerful predictor for subsequent consistent use [11]. As found earlier [4], pledgers are significantly less likely to have used a condom at first intercourse than nonpledgers (Table 4). However, pledgers are not less likely to use condoms at most recent intercourse reported in wave 3 , or in the 12 months preceding the wave 3 interviews.

Because virginity is often culturally linked only to vaginal sex, to preserve virginity, adolescents and young adults may engage in other sexual behaviors that involve exchange of fluid and are thus salient for STD acquisition. Overall, oral sex and anal sex are prevalent behaviors in this population, most commonly in conjunction with vaginal sex. Here we consider those who have oral or anal sex without vaginal sex. Amongst those who have only oral sex and/or anal sex, pledgers are over-represented. Overall, about 3\% of respondents reported oral sex with one or more partners but no vaginal sex. Although just over $2 \%$ of nonpledgers fall into this group, $13 \%$ of consistent pledgers and 5\% of inconsistent pledgers do $(p \leq .000)$. Similarly, $0.7 \%$ of nonpledgers report anal but no vaginal sex, compared with $1.2 \%$ for pledgers. Although too few females report anal but no vaginal sex, for males we find a significant difference between pledgers and nonpledgers $(p=.021)$. Specifically, slightly more than $1 \%$ of male nonpledgers report anal sex but no vaginal sex, compared with almost $3 \%$ for inconsistent pledgers and $4 \%$ for consistent pledgers. For oral sex, condom use is almost completely absent-respondents reported condom use for first oral sex for only $4 \%$ of the relationships that involved oral sex. For anal sex, condom use is also lower than for vaginal sex. Condoms were used in about $30 \%$ of relationships involving anal sex when partners had anal sex for the first time. The combination of low condom use and over-representation of pledgers provides some support for the hypothesis that this behavioral pattern is associated with greater than expected STD acquisition among pledgers, although the numbers are small and provide an insufficient basis from which to make inference.

\section{Other STD risk factors}

Here we consider additional risk factors that are known to be associated with acquisition of an STD. The first is number of sex partners. Pledgers have fewer partners than nonpledgers. Whereas the typical nonpledger male has had 2.4 
partners, male pledgers have 1.5 partners on average $(p<$ .000). The same pattern holds for females as well, 2.7 for nonpledgers and 1.9 for pledgers $(p<.000)$. Inconsistent pledgers are between these ranges (2.0 for males and females, respectively). Nor are pledgers exposed to STD risk for as long as nonpledgers. The average number of years of sexual activity, or time of exposure, is shorter for pledgers than for others. Consistent pledgers were sexually active for an average of 4.2 years, compared with inconsistent pledgers with 4.4 years and nonpledgers with 5.9 years $(p<$ $.000)$. Thus, with respect to both the number of partners and cumulative exposure, pledgers are at lower risk to acquisition of an STD than nonpledgers.

It is possible that although pledgers have fewer partners than nonpledgers, their partners might have more concurrent partners, thus putting them at enhanced risk. We test this idea indirectly, from self-report data. STD rates are in the expected direction-specifically STD rates are 7\% for those with none, and $10 \%$ for those with two or more nonmonogamous partners $(p=.002)$. But pledgers are much less likely (6\% vs. $12 \%$ ) than nonpledgers to report that their partners are nonmonogamous. Although pledgers might be less aware of their partners' nonmonogamous behavior than others, from these data there is no support for the idea that the higher-than-expected pledge STD rate is the result of high-risk partners.

\section{Health behavior}

Although those who take virginity pledges have STD rates comparable to those who do not, they are less aware of their STD status. Table 4 shows that in the year preceding the interview, pledgers were less frequently tested for and/or diagnosed with an STD than nonpledgers. Most notably, nonpledging females are almost twice as likely as females who pledged to be tested, $p \leq .000$ ). Furthermore, pledgers are significantly less likely to report seeing a doctor because they are worried about an STD $(p \leq .000)$.

\section{Discussion}

Contrary to expectations, we found no significant differences in STD infection rates between pledgers and nonpledgers, despite the fact that they transition to first sex later, have less cumulative exposure, fewer partners, and lower levels of nonmonogamous partners. Examination of the point estimates revealed small or nonexistent differences between pledgers and others, with the exception of white respondents. Advocates for abstinence-only education assert that premarital abstinence and postmarital sex are necessary and sufficient for avoiding negative consequences of sexual activity, such as STDs. This assertion collides with the realities of adolescents' and young adults' lives in several ways. First, although pledgers experience sexual debut later than others, most of them will eventually engage in premarital sex. Those who do report lower frequency of condom use at first intercourse. Those who do not are more likely to substitute oral and/or anal sex for vaginal sex.

Second, although marriage is protective against STDs, it is not perfect. Although female pledgers marry earlier, and an estimated $12 \%$ did not report any premarital sex, married pledgers test positive at the same rates as married nonpledgers. The biomarker data we have analyzed in this article cannot tell us whether pledgers had a lower risk of STD infection as young adolescents. By the end of their teenage years, at the time of wave 3 , however, these advantages, if any, have vanished. As a social policy, pledging does not appear effective in stemming STD acquisition among young adults.

Furthermore, because most adolescents eventually become sexually active during their teenage years, is it really wise to ban discussion of contraception and STD protection from sex education? Although virtually all adolescents say they learned about STDs in school [12,13], other studies have shown that many adolescents underestimate their infection risk and that they have mistaken ideas about what protects them from STDs and what does not $[14,15]$. The organizations that promote pledging and other abstinenceonly programs have been hostile to programs that combine abstinence education with information about how to prevent pregnancy and STDs for sexually active adolescents. The materials distributed by "True Love Waits" and other organizations teach adolescents that the only protection from pregnancy and STDs is abstinence. The all-or-nothing approach advocated by many abstinence-only programs may create additional barriers to knowledge and protection for adolescents. For example, the emphasis on virginity may encourage adolescents to limit their sexual activity to noncoital behaviors, which may nevertheless expose them to risks of infection. In this context, it is important to know that pledgers are less likely than nonpledgers to be tested for STDs, and to have ever seen a doctor because they are worried about an STD. If STDs were more likely to go untreated among pledgers, higher STD prevalence may result even in the presence of lower incidence rates.

Systematic and rigorous evaluation of the health impact of abstinence-only programs has rarely been undertaken [16]. The results presented in this article show that a careful evaluation should accompany the generous federal and state funds that abstinence-only programs have enjoyed. At least for one such program, pledging, our findings suggest that a short-term evaluation of the behavioral impact of sex education programs is not sufficient to predict the longer-term health impact on STD rates.

\section{Acknowledgments}

This research uses data from Add Health, a program project designed by J. Richard Udry, Peter S. Bearman, and Kathleen Mullan Harris, and funded by a grant P01HD31921 from the National Institute of Child Health and 
Human Development, with cooperative funding from 17 other agencies. Special acknowledgment is due Ronald R. Rindfuss and Barbara Entwisle for assistance in the original design. Persons interested in obtaining data files from Add Health should contact Add Health, Carolina Population Center, 123 W. Franklin Street, Chapel Hill, NC 275162524. The authors thank two anonymous reviewers for their helpful comments. Authorship is in reverse alphabetical order. Both authors contributed equally to this article.

\section{References}

[1] Centers for Disease Control and Prevention. Tracking the hidden epidemic: trends in STDs in the United States [cited 2004 Jul 14]. Available from: www.cdc.gov/nchstp/od/news/RevBrochure1.pdf.htm.

[2] Centers for Disease Control and Prevention. Sexually Transmitted Disease Surveillance, 2001. Altlanta, GA: Department of Health and Human Services, CDC, 2002.

[3] Weinstock H, Berman S, Cates W Jr. Sexually transmitted diseases among American youth: incidence and prevalence estimates, 2000. Perspect Sex Reprod Health 2004;36:6-10.

[4] Bearman P, Brückner H. Promising the future: abstinence pledges and the transition to first intercourse. Am J Sociol 2001;106:859912.

[5] Add Health Biomarker Team. Biomarkers in Wave III of the Add Health Study [cited 2004 Jul 14]. Available from: http://www.cpc. unc.edu/addhealth/files/biomark.pdf.
[6] Carolina Population Center. Wave III data documentation [cited 2004 Jul 14]. Available from: http://www.cpc.unc.edu/projects/addhealth/ codebooks/wave3.

[7] Chantala K, Kalsbeek WD, Andraca E. Non-response in Wave III of the Add Health study [2004 Jun 28]. Available from: http://www.cpc. unc.edu/projects/addhealth/files/W3nonres.pdf.

[8] Chantala, K, Tabo J. Strategies to perform design-based analysis using the Add Health Data [cited 2004 Jul 14]. Available from: www.cpc.unc.edu/projects/addhealth/files/weight1.pdf.

[9] Stata Corp. Stata Statistical Software: Release 8.0. College Station, TX: Stata Corporation, 2003.

[10] Miller WC, Ford CA, Hobbs MM, et al. Prevalence of chlamydial and gonococcal infections among young adults. JAMA 2004;292:801-2.

[11] Shafii T, Stovel K, Davis R, Holmes KK. Is condom use habitforming? Condom use at sexual debut and subsequent condom use. Sex Transm Dis 2004;31(6):366-72.

[12] Lane T. Despite having received relevant education, youth lack knowledge of STDs. Perspect Sex Reprod Health 2003;35:51.

[13] Brückner H, Bearman P. Dating behavior and sexual activity among young adolescents. In: Albert B, Brown S, Flanigan CM, eds. 14 and Younger: The Sexual Behavior of Young Adolescents. Washington, DC: Campaign to Prevent Teen Pregnancy 2003:31-56.

[14] Kaiser Family Foundation. Seventeen. A series of national surveys of teens about sex: birth control and protection [cited 2004 Dec 6]. Available from: www.kff.org/entpartnerships/phip070604pkg.cfm.

[15] Kaiser Family Foundation. Seventeen. A series of national surveys of teens about sex: sexually transmitted diseases [cited 2004 Dec 6]. Available from: www.kff.org/entpartnerships/seventeen_surveys.cfm.

[16] Kirby D. Emerging Answers: Research Findings on Programs to Reduce Teen Pregnancy. Washington, DC: National Campaign to Prevent Teen Pregnancy, 2001. 